\title{
Fingolimod: A Disease-Modifier Drug in a Mouse Model of Amyotrophic Lateral Sclerosis
}

\author{
Rosa Luisa Potenza ${ }^{1}$ - Roberta De Simone ${ }^{2} \cdot$ Monica Armida $^{1} \cdot$ Valentina Mazziotti $^{2}$. \\ Antonella Pèzzola ${ }^{1} \cdot$ Patrizia Popoli $^{1} \cdot$ Luisa Minghetti $^{2}$
}

Published online: 25 July 2016

(C) The American Society for Experimental NeuroTherapeutics, Inc. 2016
Key Words Amyotrophic lateral sclerosis (ALS) . Fingolimod $\cdot$ FTY720 $\cdot \mathrm{mSOD}^{\mathrm{G} 93 \mathrm{~A}}$ mice . neuroinflammation

\section{Introduction}

Amyotrophic lateral sclerosis (ALS) is an incurable disease characterized by progressive degeneration of motor neurons in spinal cord and motor cortex [1]. ALS is currently considered a multifactorial disease, which partially explains the failure to develop effective therapeutic approaches. To date, despite the many drugs tested in phase II and phase III clinical trials, riluzole remains the only treatment approved for ALS, and, unfortunately, it has only limited effects.

A growing body of evidence suggests that, besides many other mechanisms (e.g., excitotoxicity, oxidative stress, toxicity by protein misfolding, and mitochondrial dysfunction, see [2]), neuroinflammation and immune reaction are pivotal features both in patients with ALS and in animal models [3-5].

Malfunction of both innate and adaptive immune system can actively influence disease progression in animal models and in patients with familial and sporadic ALS [6, 7]. Substantial numbers of infiltrating $\mathrm{T}$ cells and macrophages are found in the spinal cord of patients with ALS [8-10]; the majority of these migrating cells are described as $\mathrm{T}$ helper (Th) and $\mathrm{T}$ suppressor/cytotoxic cells [11], whereas a decreased number of regulatory $\mathrm{T}$ cells (Tregs) was observed in mice and patients with ALS during the rapidly progressing phase of the disease [12].

Microglia, the resident immune cells of the central nervous system (CNS), play a pivotal role in ALS pathology; the presence of activated microglial cells, in regions of motor neuron damage, was reported both in patients with ALS and in mouse 
models of ALS where microgliosis is already present prior to disease onset [13-15].

It is now well accepted that during disease progression, a multiphasic immune response occurs in ALS whereby a shift from type II (T2) beneficial immune responses (involving M2 macrophages/microglia, Th2 responses, and Tregs) to a neurotoxic type I (T1) response (involving M1 microglia and Th1 responses) takes place [5]. Hence, microglia and lymphocytes, depending on their phenotype and activation status, and according to the stage of the disease, can have both neurotoxic and neuroprotective functions in ALS [16, 17].

Fingolimod (FTY720), the first approved oral therapy for multiple sclerosis, is a sphingosine 1-phosphate receptor agonist. Unlike conventional immunosuppressive drugs, fingolimod does not inhibit T- or B-cell activation [18, 19], but it reduces the migration of pathogenic lymphocytes into the CNS ("indirect" CNS mechanism). Fingolimod also increases the number of circulating Tregs, eventually causing a redistribution rather than a depletion of lymphocytes [20].

After systemic administration fingolimod readily accesses the CNS where endogenous sphingosine kinases generate the active form of the drug and where it exerts multiple effects acting on resident cells ("direct" CNS mechanism) [21], such as promoting the neuroprotective effects of microglia through a downregulation of proinflammatory cytokine production [22], and protecting neurons against excitotoxic death by inhibiting p38 mitogen-activated protein kinase activation [23].

On the basis of the above considerations, fingolimod can represent a promising therapeutic approach for ALS as it has the potential to impact simultaneously on different pathogenic mechanisms of the disease, including microglial activation, excitotoxicity, and peripheral immune response. The relevance of such an approach in ALS is testified to by the recent approval, in the USA, of a phase IIA clinical trial of fingolimod in patients with ALS. Although the wish of a rapid clinical translation of a promising treatment is fully understandable, the lack of preclinical investigations raises some concerns. First, without mechanistic studies it is difficult to foresee the real therapeutic potential of the approach; second, as immune activation and neuroinflammation in ALS are multifaceted, with both positive and negative aspects, their role change substantially during the course of the disease and the outcome of a treatment might vary dramatically according to the stage and rate of progression.

The present study was designed to determine whether chronic treatment with fingolimod is able to extend the survival and improve the phenotype of mutant superoxide dismutase 1 (SOD1) ${ }^{\mathrm{G} 93 \mathrm{~A}}$ mice, a well-characterized mouse model of ALS, and to establish whether fingolimod effects correlate with a modulation of neuroinflammatory parameters in motor cortex and spinal cord of ALS mice.

\section{Materials and Methods}

\section{Animals}

Transgenic mice expressing high copy number of G93A mutant form of human SOD1 (mSOD1 ${ }^{\mathrm{G} 93 \mathrm{~A}}-\mathrm{B} 6 . \mathrm{Cg}-$ $\left.\operatorname{Tg}\left(\mathrm{SOD} 1{ }^{*} \mathrm{G} 93 \mathrm{~A}\right) 1 \mathrm{Gur} / \mathrm{J}\right)$, and wild-type (WT; age-matched nontransgenic littermates) mice were originally obtained from Jackson Laboratories (Bar Harbor, ME, USA) and bred in our indoor animal facility. Transgenic hemizygous $\mathrm{mSOD} 1^{\mathrm{G} 93 \mathrm{~A}}$ males were crossbred with $\mathrm{C} 57 \mathrm{BL} / 6$ females, both maintained on the C57BL/6 genetic background. Transgenic progeny were genotyped by analyzing DNA tissue extracts from tail tips, using a standard polymerase chain reaction (PCR) protocol with specific primers for human SOD1: SOD forward 5' CATCAGCCCTAATCCATCTGA - 3'; SOD reverse 5' CGCGACTAACAATCAAAGTGA - 3'; interleukin (IL)-2 forward 5'-TAGCCACAGAATTGAAAGATCT-3'; IL-2 reverse 5'-GTAGGTGGAAATTCTAGCATCATCC - 3'.

The animals were kept under standardized temperature, humidity, and lighting conditions, with free access to water and food (standard pellets). Animal care and use followed the European Directive 2010/63/EU, adopted by Council of the European Union for animal experiments, and adequate measures were taken to minimize pain or discomfort. When mice showed substantial motor impairment pellets soaked in normal water were supplied inside the cage. The experimental protocol was approved by the Italian Ministry of Health.

\section{Treatments}

As the majority of patients with ALS are sporadic, and treatment can only be started at the time of diagnosis, $\mathrm{mSOD}^{\mathrm{G} 93 \mathrm{~A}}$ mice were treated starting from the onset of motor symptoms (i.e., from around 16-17 weeks of age) until the end stage of disease. Fingolimod (Sigma-Aldrich, St. Louis, MO, USA) was administered intraperitoneally 3 times weekly at doses $(0.1$ or $1 \mathrm{mg} / \mathrm{kg})$ that have been shown to be protective in other animal models of neurodegeneration [24, 25].

We enrolled the following animals per experimental group: WT vehicle $=5$ female and 5 male; mSOD 1 vehicle $=8$ females and 11 males; mSOD1 fingolimod $0.1=6$ females and 6 males; mSOD1 fingolimod $1=5$ females and 4 males.

Drug- and vehicle-treated animals were monitored for survival (primary endpoint). Body weight, motor function, and neurological score were considered as secondary endpoints. The expression of genes related to neuroinflammation was analyzed in motor cortex and spinal cords of end-stage mice.

To get insight into the time course of the effects of fingolimod, another group of $\mathrm{mSOD} 1{ }^{\mathrm{G} 93 \mathrm{~A}}$ mice ( $n=4$ /group balanced for sex) was treated from the onset of symptoms with the lower dose of fingolimod $(0.1 \mathrm{mg} / \mathrm{kg})$ and tissues were collected after 2 weeks of fingolimod administration for 
molecular analysis. The experimental groups were sex balanced; a group of vehicle-treated WT mice $(n=8)$ were also analyzed for internal control.

These animals were subjected to all behavioral tests used in preclinical trials in order to obtain experimental groups comparable for stress, manipulation, and physical exercise.

\section{Body Weight}

Body weight was measured for each animal 3 times weekly (from 9 a.m. to 12 a.m., to avoid diurnal variations) using a digital scale.

\section{Motor Function Testing}

Motor function and coordination were tested using an accelerated rotarod device (Columbus Instruments, Columbus, $\mathrm{OH}$, USA). At 15 weeks of age, mice were trained for 2 days to become acquainted with the rotarod. In the training sessions a fixed speed protocol $(20 \mathrm{rpm})$ with a cut off of $180 \mathrm{~s}$ was applied. The testing began at 16 weeks of age using a ramped accelerating program: the animals were positioned on the rotating bar, time was started, and the rod was accelerated at a constant rate from $8 \mathrm{rpm}$ to $32 \mathrm{rpm}$ for a maximum of $180 \mathrm{~s}$. Mice were given 3 consecutive trials (10 min intervals), and for each animal the longest latency to fall was recorded as a measure of the motor function competence. Rotarod testing was performed once a week until the animals reached the preestablished minimum performance $(5 \mathrm{~s})$.

\section{Neurological Score Evaluation}

Neurological evaluation of mSOD $1^{\mathrm{G} 93 \mathrm{~A}}$ mice was assessed (3 times weekly from 15 weeks of age) employing the ALS Therapy Development Institute neurological score system proposed by Scott et al. [26]: $0=$ full extension of hindlimbs away from lateral midline when mouse is suspended by its tail; 1 = collapse or partial collapse of leg extension towards lateral midline (weakness) or trembling of hindlimbs during tail suspension; 2 = during walking any part of foot is dragging along table (walk with enlarged posterior train); 3 = rigid paralysis or minimal joint movement, foot not being used for forward motion; $4=$ mouse cannot right itself within $30 \mathrm{~s}$ from either side. For the data analysis, dead mice were included in category " 4 ".

\section{Determination of Disease Onset, End-Stage, and Survival}

Disease onset was defined as the time at which the rotarod performance of the $\mathrm{mSOD} 1^{\mathrm{G} 93 \mathrm{~A}}$ mice was significantly reduced with respect to WT mice.

The survival time was considered as the actual age of death or the time (defined as end stage), when mice were sacrificed because of the loss of the ability to right themselves within $30 \mathrm{~s}$ after having been placed on their sides, according to established guidelines for drug testing in ALS mouse models [27]. WT animals were sacrificed when the last transgenic mice died.

\section{Quantitative Real-Time PCR}

To characterize the mechanisms of actions of fingolimod in ALS mice, expression of genes related to neuroinflammation were analyzed in motor cortex and spinal cords homogenates of $\mathrm{mSOD} 1^{\mathrm{G} 93 \mathrm{~A}}$ mice treated with fingolimod or vehicle. Experimental groups were sex balanced. A group of vehicletreated WT mice $(n=8)$ was also analyzed as internal controls.

Total RNA $(1 \mu \mathrm{g})$ from each sample was transcribed into complementary DNA using the real-time PCR Superscript III kit (Invitrogen, Eugene, OR, USA), according to the manufacturer's instructions. Real-time PCR was performed on the reverse transcription products with a SensiMix SYBR Kit [Bioline, London, UK; for hypoxanthine guanine phosphoribosyl transferase (HPRT), inducible nitric oxide synthase (iNOS), IL-1 $\beta$, IL-10, and arginase 1 (Arg-1) mRNA expression] or with TaqMan [for HPRT, CD11b, FoxP3, and brain-derived neurotrophic factor (BDNF)] using an ABI Prism 7500 Sequence Detection System (Applied Biosystems, Foster City, CA, USA). Primers for HPRT (Mn.PT.39a22214828), CD11b (Mn.PT.58.9189361), and FoxP3 (Mn.PT.58.30761183) were from Integrated DNA Technologies (IDT, TEMA Ricerca Bologna, Italy); primers for BDNF (Mn.0423060711) were from Applied Biosystems. Primer sequences for HPRT iNOS, IL-1 $\beta$, Arg-1, and IL-10 were from Integrated DNA Technologies; accession numbers are as follows: 1) HPRT (NM_013556): forward 5'-CAGGCCAGACTTTG-TTGGAT-3'; reverse 5'TTGCGCTCATC-TTAGGCTTT-3'; 2) IL-1 $\beta$ (NM_008361): forward 5'-CGACAAAATACCTGTGGCCT-3', reverse 5'TTCTTTGGGTATTCCTTGGG-3'; 3) iNOS (NM_010927): forward 5'-CAGCTGGGCTGTACAAACCTT-3', reverse 5'CATTGGAAGTGAA GCGTTTCG-3'; 4) IL-10 (NM_010548): forward 5'-TTAAGCTGTTTCCATTGGGG3', reverse 5'-AAGTGTGGCCAGCCTTAGAA-3'; 5) Arg-1 (NM_007482): forward 5'-GGAAAGCCAATGAA GAGCTG-3', reverse 5'-AACACTCCCCTGACAACCAG-3'.

Annealing temperature was $60{ }^{\circ} \mathrm{C}$ for all the primer pairs listed. All samples were run in triplicate, and each PCR well contained $20 \mu \mathrm{l}$ as a final volume of reaction, including $2 \mu \mathrm{l}$ complementary DNA corresponding to approximately $60 \mathrm{ng}$ total RNA, $750 \mathrm{nM}$ of each primer, and $10 \mu \mathrm{l}$ PCR master mix. Thermal cycling conditions were as follows: 1 cycle at $95{ }^{\circ} \mathrm{C}$ for $10 \mathrm{~min} ; 40$ cycles at $95^{\circ} \mathrm{C}$ for $15 \mathrm{~s}$, and $60{ }^{\circ} \mathrm{C}$ for $1 \mathrm{~min}$. The relative expression level of each mRNA was calculated using the $\Delta \Delta \mathrm{Ct}$ method normalized to HPRT and relative to the control samples. The amplification specificity was verified by melting curve analyses. 


\section{Statistics}

Kaplan-Meier survival analysis and log-rank (Mantel-Cox) were used for survival and neurological onset comparisons.

Body weight, rotarod performance, and neurological score data were statistically analyzed with 2-way analysis of variance for repeated measures (time) and different groups (treatment).

Gene expression results (mean \pm SEM of 4 animals per group or as indicated in the figure legends) are given as fold increase over control group, either WT or vehicle-treated $\mathrm{mSOD} 1^{\mathrm{G} 93 \mathrm{~A}}$ mice, as indicated in figure legends. Statistical significance was evaluated using Student's $t$ test; $p<0.05$ was accepted as statistically significant.

\section{Results}

\section{Fingolimod Delays Neurological Deficits and Extends Lifespan of Symptomatic mSOD1 ${ }^{\text {G93A }}$ Mice}

To reduce the variability among cohorts, each experimental group was littermate and age matched, balanced for sex, and animals dead as a result of non-ALS-related causes were excluded from study analyses.

As expected, $\mathrm{mSOD} 1^{\mathrm{G} 93 \mathrm{~A}}$ mice showed a gradual decrease in body weight (Fig. 1A), and a significant worsening of motor performance in the rotarod device started from 114 days of age (Fig. 1B). Starting from this time point, defined as motor disease onset, animals were treated with fingolimod (at 2 different doses) or vehicle.

Both body weight and motor performance were unaffected by chronic administration of fingolimod from motor deficit onset until end stage (Fig. 1A, B).

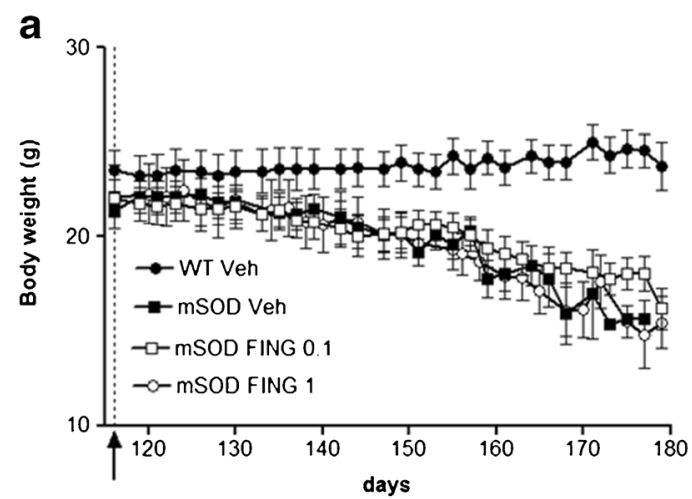

Fig. 1 Fingolimod (FING) had no significant effect on rotarod performance and body weight in $\mathrm{mSOD} 1^{\mathrm{G} 93 \mathrm{~A}}$ mice. Fingolimod $(0.1$ and $1 \mathrm{mg} / \mathrm{kg}$ ) was injected i.p. 3 times weekly from the onset of symptoms until the end of life. (A) Body weight was recorded 3 times a week. (B) Motor function was tested once a week using an accelerated rotarod device, as defined in the "Materials and Methods". Values (mean
Conversely, fingolimod, at both tested doses, significantly ameliorated neurological deficits. As shown in Fig. 2A, mSOD1 ${ }^{\mathrm{G} 93 \mathrm{~A}}$ mice in the fingolimod groups exhibited significantly reduced neurological scores compared with the vehicle-treated group throughout the trial; 2-way analysis of variance demonstrated a significant time $\times$ treatment interaction in neurological scoring $[\mathrm{F}(24,481)=1.59$; $p<0.05]$, indicating that the pharmacological effect of fingolimod changes during the progression of the disease. Post-hoc Tukey's multiple comparisons test showed that the pharmacological effects of the 2 doses of fingolimod was comparable, suggesting the occurrence of a ceiling effect at a dose of $0.1 \mathrm{mg} / \mathrm{kg}$. When administered from early symptomatic phase of the disease, fingolimod significantly delayed neurological onset (vehicle median onset $=136$ days; fingolimod $0.1 \mathrm{mg} / \mathrm{kg}=$ 145 days; $p<0.001, \log$-rank test; Fig. 2B); at the final stage of the disease, mSOD $1^{\mathrm{G} 93 \mathrm{~A}}$ mice treated with vehicle displayed a median onset for score 4 at 164 days, whereas in drug-treated mSOD1 mice the score of 4 arose, on average, at 181 days $(p<0.01$, log-rank test; Fig. 2 C).

Most importantly, fingolimod significantly prolonged the lifespan of $\mathrm{mSOD} 1^{\mathrm{G} 93 \mathrm{~A}}$ mice at both doses $(p<0.01$, log-rank test). As shown in Fig. 3, the median survival time for the control group was 160 days, whereas $50 \%$ of animals treated with fingolimod were still alive 2 weeks later (median survival $=175.5$ and 171 days for 0.1 and $1 \mathrm{mg} / \mathrm{kg}$ fingolimod, respectively). Again, the effect of fingolimod was not dose dependent.

\section{Fingolimod Modulates the Neuroinflammatory Response in $\mathrm{mSOD1}^{\mathrm{G93A}}$ Mice}

To gain some insights into the molecular mechanisms affected by fingolimod treatment, we analyzed the

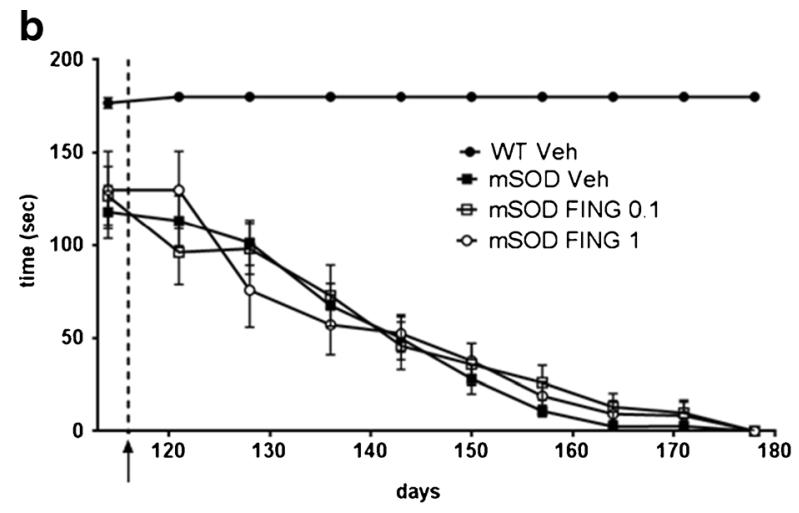

\pm SEM) for body weight and rotarod performance of drug-treated $\mathrm{mSOD} 1^{\mathrm{G} 93 \mathrm{~A}}$ mice (FING $0.1 \mathrm{mg} / \mathrm{kg} n=12$; FING $1 \mathrm{mg} / \mathrm{kg} n=9$ ) and saline-treated $\mathrm{mSOD} 1^{\mathrm{G} 93 \mathrm{~A}}$ [vehicle (Veh) $n=19$ ] were indistinguishable ( $p>0.05 v s$ ALS-vehicle group; 2-way analysis of variance). The start of fingolimod treatment is indicated by black arrows 


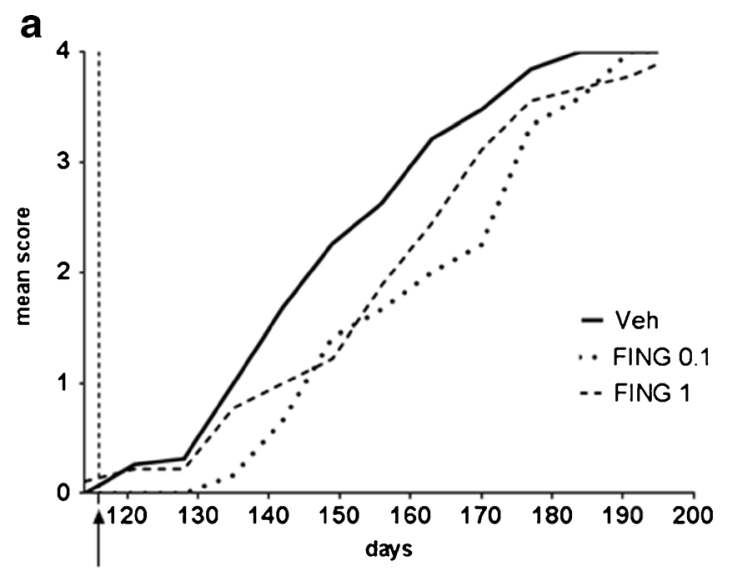

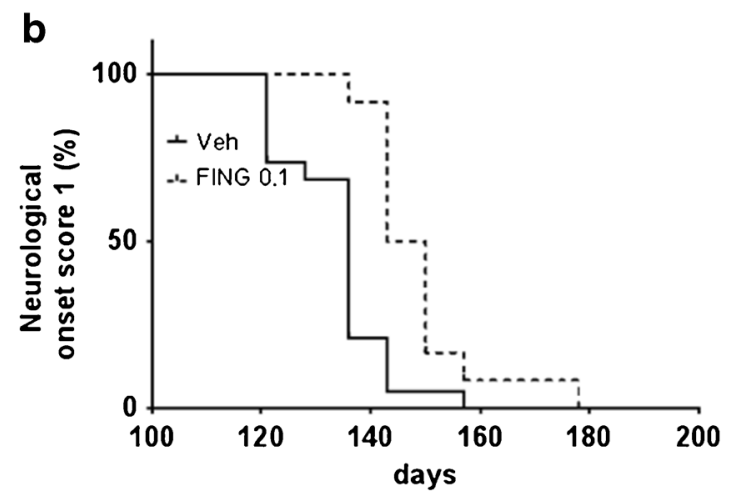

Fig. 2 Fingolimod (FING) delays neurological deficits in $\mathrm{mSOD} 1^{\mathrm{G} 93 \mathrm{~A}}$ mice. (A) Neurological mean score indicates a significant attenuation in disease progression in mice treated with fingolimod $(0.1 \mathrm{mg} / \mathrm{kg}$ and $1 \mathrm{mg} /$ $\mathrm{kg}$ ) compared with saline-treated controls ( $p<0.05,2$-way analysis of variance with post hoc Tukey's multiple comparisons). Fingolimod treatment $(0.1 \mathrm{mg} / \mathrm{kg})$ significantly delayed neurological onset of $(\mathbf{B})$ score 1

expression of some genes relevant to neuroinflammatory and immune processes; mRNA levels for the selected genes were assessed by real-time PCR in the lumbar and cervical spinal cord and in the motor cortex of mSOD $1^{\text {G93A }}$ mice treated with vehicle or with the lowest dose of fingolimod $(0.1 \mathrm{mg} / \mathrm{kg})$ for 2 weeks or until the end stage of disease. The 2-week treatment was chosen as an early time point at which the amelioration of neurological deficits could already be detected.

First, we focused on CD11b, a microglia-macrophagespecific activation marker, and FoxP3, a transcription factor required for Treg functions that have been associated with stable protective phase (T2) of the disease in ALS mice [28]. Compared with WT mice, 2 weeks after the appearance of motor symptoms, $\mathrm{mSOD} 1^{\mathrm{G} 93 \mathrm{~A}}$ mice exhibited increased mRNA levels for CD11b in the lumbar and cervical spinal cord, while in the cortex, CD11b mRNA levels were not yet altered (Table 1). After 2 weeks of treatment with $0.1 \mathrm{mg} / \mathrm{kg}$ fingolimod (Fig. 4, light bars), the mRNA for CD11b in all analyzed regions was unaltered compared with vehicle-treated

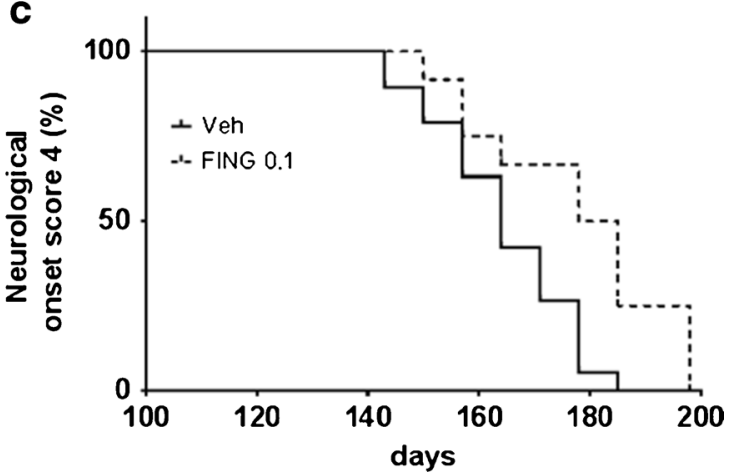

[vehicle $($ Veh $)$ median onset $=136$ days, FING $0.1=145$ days $(* * * p<0.001)$ log-rank test] and $(\mathbf{C})$ score 4 (Veh median onset $=164$ days, FING $0.1=181$ days, $* * p<0.01$ log-rank test) in drug-treated $\mathrm{mSOD} 1^{\mathrm{G} 93 \mathrm{~A}}$ mice compared with mutant littermates treated with vehicle. The start of fingolimod treatment is indicated by the black arrow

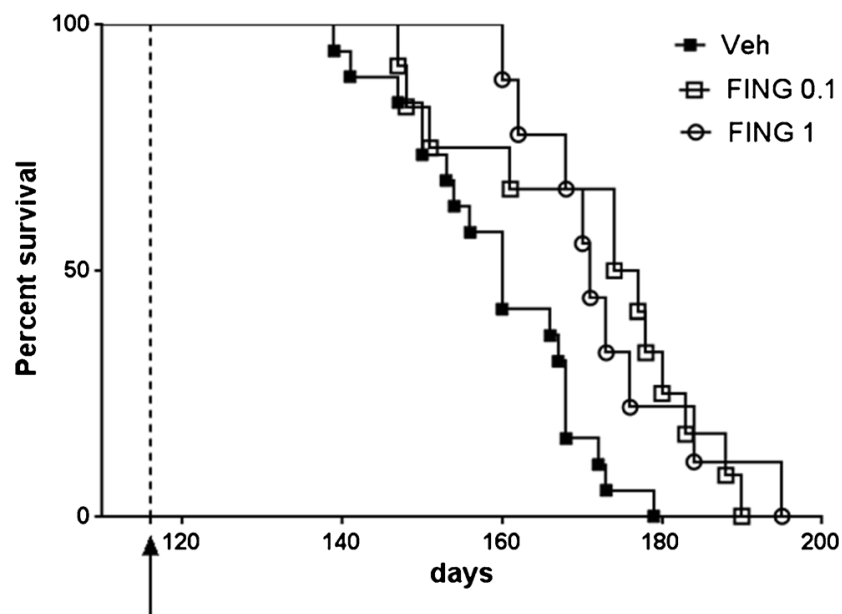

Fig. 3 Fingolimod (FING) significantly extends survival of mSOD $1^{\mathrm{G} 93 \mathrm{~A}}$ mice at both doses tested. Cumulative probability of survival in mutant superoxide dismutase (mSOD) $1{ }^{\mathrm{G} 93 \mathrm{~A}}$ transgenic mice treated with fingolimod (FING), at $0.1 \mathrm{mg} / \mathrm{kg}(n=12)$ and $1 \mathrm{mg} / \mathrm{kg}(n=9)$, or vehicle (Veh) $(n=19)$ injected intraperitoneally 3 times weekly. Survival was significantly prolonged in drug-treated $\mathrm{mSOD} 1^{\mathrm{G} 93 \mathrm{~A}}$ mice compared with mutant littermates treated with vehicle (Veh median survival $=160$ days, FING $0.1 \mathrm{mg} / \mathrm{kg}=175.5$ days, FING $1 \mathrm{mg} / \mathrm{kg}=171$ days; $* * p<0.01$ log-rank test) 
Table 1 Neuroinflammatory response in $\mathrm{mSOD} 1^{\mathrm{G} 93 \mathrm{~A}}$ mice 2 weeks after motor deficit onset

\begin{tabular}{llll}
\hline Gene & Cortex & $\begin{array}{l}\text { Cervical spinal } \\
\text { cord }\end{array}$ & $\begin{array}{l}\text { Lumbar spinal } \\
\text { cord }\end{array}$ \\
& $\begin{array}{l}\text { Fold change } \\
\text { mSOD1 } \\
\text { wild type }\end{array}$ & & \\
\hline CD11b & $0.99 \pm 0.11$ & $3.88 \pm 0.59^{*}$ & $2.09 \pm 0.17^{*}$ \\
FoxP3 & $15.59 \pm 2.13^{*}$ & $22.68 \pm 3.13^{*}$ & $5.92 \pm 1.02^{*}$ \\
iNOS & $0.47 \pm 0.09^{*}$ & $1.02 \pm 0.13$ & $0.53 \pm 0.19^{*}$ \\
Il1 $\beta$ & $0.26 \pm 0.12^{*}$ & $2.35 \pm 0.19^{*}$ & $1.31 \pm 0.54$ \\
Arg1 & $0.68 \pm 0.21$ & $0.11 \pm 0.02^{*}$ & $11.02 \pm 3.88^{*}$ \\
Il10 & $0.28 \pm 0.08^{*}$ & $0.91 \pm 0.57$ & $0.38 \pm 0.15^{*}$ \\
\hline
\end{tabular}

Expression of each gene is presented as fold-change over the expression measured in the same region of wild-type mice, taken as 1 . The relative expression level of each mRNA was calculated using the ${ }^{2 \Delta} \mathrm{Ct}$ method, normalizing to hypoxanthine guanine phosphoribosyl transferase, as detailed in the "Materials and Methods" section. Data are mean \pm SEM, $n=4-8$

$* p<0.05$

$\mathrm{mSOD} 1^{\mathrm{G} 93 \mathrm{~A}}$ mice. At the end stage of the disease (Fig. 4, dark bars), chronic administration of fingolimod induced a significant reduction of CD11b mRNA in lumbar spinal cord and motor cortex, while in cervical spinal cord the expression was unaffected, suggesting a regionspecific downregulation of microglial activation.

Two weeks after motor symptom onset, FoxP3 mRNA levels in $\mathrm{mSOD} 1^{\mathrm{G} 93 \mathrm{~A}}$ mice were increased in all 3 regions compared with WT mice (Table 1), with the highest expression detected at the level of the cervical spinal cord. After 2 weeks, fingolimod administration significantly increased FoxP3 mRNA levels only in the cervical spinal cord compared with vehicle-treated mSOD1 ${ }^{\mathrm{G} 93 \mathrm{~A}}$ mice (Fig. 4, light bars); at end stage drug-treated $\mathrm{mSOD} 1^{\mathrm{G} 93 \mathrm{~A}}$ mice showed higher levels of FoxP3 mRNA than mSOD1 ${ }^{\mathrm{G} 93 \mathrm{~A}}$ mice treated with vehicle in all 3 analyzed regions (dark bars).

In order to investigate some functional indicators of the ongoing immune response, we analyzed the expression of genes associated with either the so-called M1 (iNOS, IL-1 $\beta$ ) or M2 (Arg-1, IL-10) microglial phenotypes in the 3 regions and at the same time points as described above.

Compared with WT mice, 2 weeks after the appearance of motor symptoms, $\mathrm{mSOD} 1^{\mathrm{G} 93 \mathrm{~A}}$ mice exhibited significant alterations in the levels of expression of all 4 genes, in a region-specific manner (Table 1). In particular, the cortex was characterized by an overall decrease of gene expression, whereas there were skewed M1- and M2-like immune responses in the cervical and lumbar spinal cord. After 2 weeks of fingolimod treatment (Fig. 5, light bars), in the lumbar spinal cord, mRNA levels of all 4 genes were reduced compared with
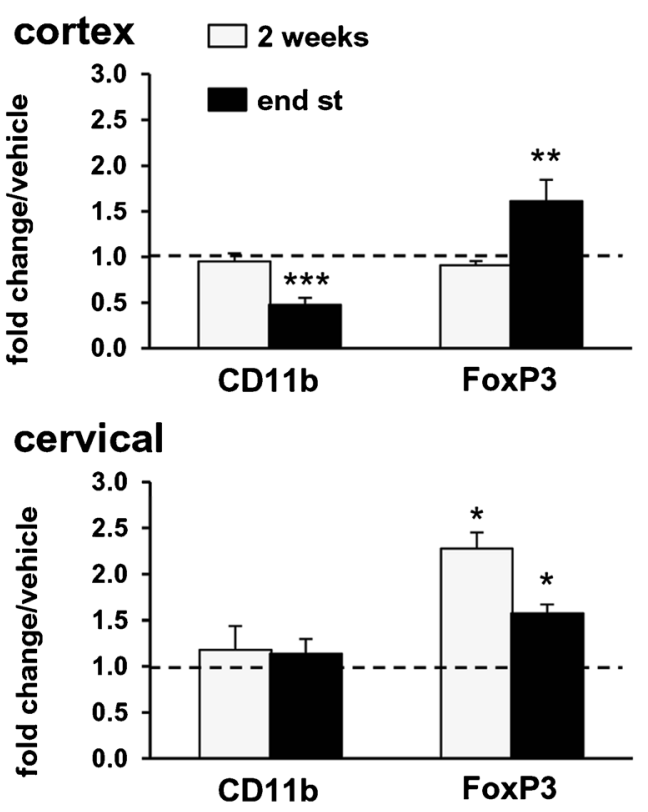

lumbar

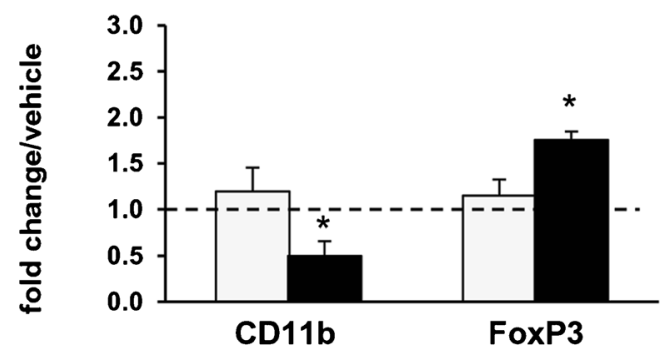

Fig. 4 Fingolimod modulates the expression of CD11b and FoxP3 transcripts in the spinal cord and cortex of $\mathrm{mSOD} 1^{\mathrm{G} 93 \mathrm{~A}}$ mice. Real-time polymerase chain reactions were performed with mRNAs extracted from motor cortex, cervical and lumbar spinal cords of $\mathrm{mSOD} 1^{\mathrm{G} 93 \mathrm{~A}}$ mice that were treated, starting from the onset of motor symptoms, with fingolimod $(0.1 \mathrm{mg} / \mathrm{kg})$ or vehicle for 2 weeks or until the end stage (end st) of the disease. Expression of each gene is presented as fold change over the expression measured in vehicle-treated animals, taken as 1 (indicated by a dotted line), for each brain region examined. After 2 weeks of treatment (light bars), CD11b mRNA was unaltered in all analyzed regions. At the end stage of the disease (dark bars), fingolimod induced a significant reduction of CD11b mRNA in lumbar spinal cord and motor cortex but not in cervical spinal cord. After 2 weeks of fingolimod administration, FoxP3 mRNA levels were significantly increased only in the cervical spinal cord $(2.3 \pm 0.2$-fold increase $v s$ vehicle-treated animals); after chronic administration of fingolimod (dark bars) FoxP3 mRNA levels were increased in all 3 regions. The relative expression level of each mRNA was calculated using the ${ }^{2 \Delta} \mathrm{Ct}$ method, normalized to hypoxanthine guanine phosphoribosyl transferase, as detailed in the "Materials and Methods" section. Data are mean \pm SEM, $n=4-6$; $* p<0.05, * * p<0.01 ; * * * p<0.001$

vehicle-treated animals; in the cervical region, no significant alterations in mRNA levels for the 4 genes were observed; in motor cortex, iNOS expression was not affected, while mRNA levels of IL-1 $\beta$, Arg-1, and IL-10 were increased compared with vehicle-treated $\mathrm{mSOD} 1^{\mathrm{G} 93 \mathrm{~A}}$ mice. 

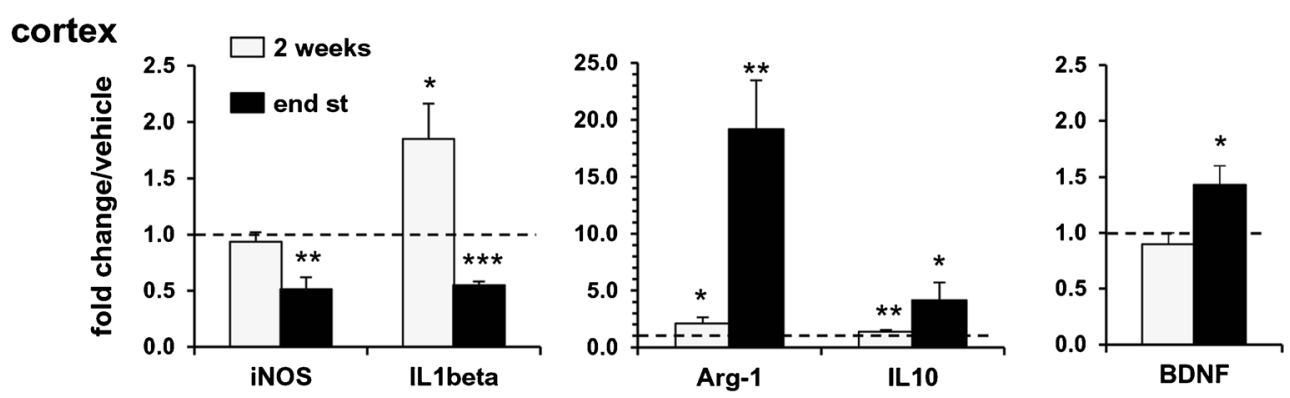

\section{cervical}
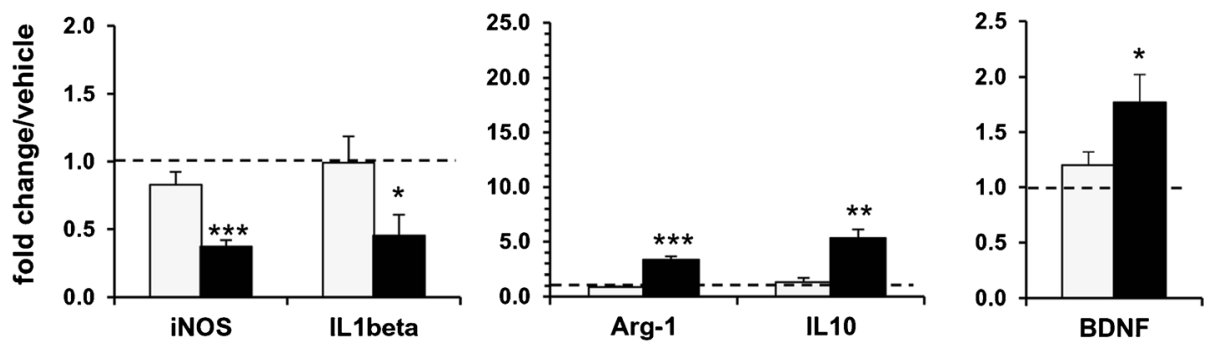

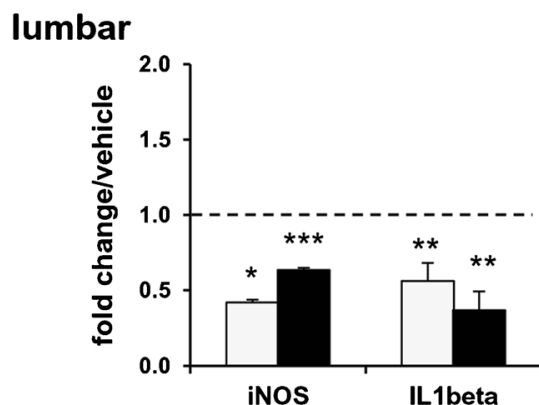

Fig. 5 Fingolimod modulates M1 and M2 mRNA markers in $\mathrm{mSOD} 1^{\mathrm{G} 93 \mathrm{~A}}$ mice. The expression profiles of inducible nitric oxide synthase (iNOS), interleukin (IL)-1 $\beta$, arginase (Arg)-1, IL-10, and brain-derived neurotrophic factor (BDNF) were examined by real-time polymerase chain reaction in motor cortex, cervical and lumbar spinal cords of $\mathrm{mSOD} 1^{\mathrm{G} 93 \mathrm{~A}}$ mice at the same time points as described in Fig. 4 (2 weeks: light bars; end stage of disease: dark bars). As for Fig. 4, expression of each gene is presented as fold change over the expression measured in vehicle-treated animals, taken as 1 (indicated in the graphs by a dotted line), for each brain region examined. After 2 weeks of fingolimod treatment (light bars), mRNA levels of M1 (iNOS, IL-1 $\beta$ )
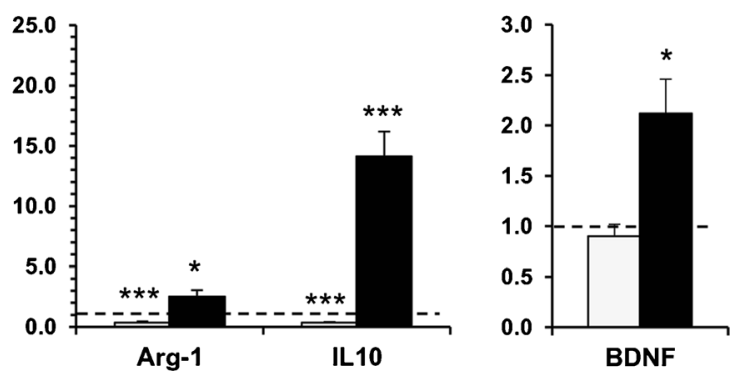

and M2 (Arg-1, IL-10) markers were reduced in the lumbar spinal cord, while no differences were observed in the cervical region. In motor cortex, fingolimod did not affect iNOS mRNA, while mRNA levels of IL$1 \beta$, Arg- 1 , and IL-10 were increased. In all 3 regions, BDNF expression was not affected. After chronic treatment (dark bars), iNOS and IL-1 $\beta$ mRNA levels were substantially decreased in spinal cord regions and in the motor cortex. Arg-1, IL-10, and BDNF were significantly upregulated in all 3 regions of drug-treated mice respect to vehicle group. Data, analyzed by the $2^{\Delta \mathrm{Ct}}$ method, are expressed as mean \pm SEM $(n=4-6)$; $* p<0.05, * * p<0.01 ; p<0.001$
At the final stage of disease, after chronic treatment with fingolimod, iNOS and IL- $1 \beta$ mRNA levels were substantially decreased in both spinal cord regions and in the motor cortex when compared with vehicle-treated mSOD $1^{\mathrm{G} 93 \mathrm{~A}}$ mice (Fig. 5, dark bars). The antiinflammatory M2 markers Arg-1 and IL-10 were both significantly upregulated in all 3 regions of drug-treated mice with respect to vehicle group (Fig. 5, dark bars), suggesting a polarization of the immune response towards a M2 type, dominated by IL-10 and Arg-1 overexpression. Interestingly, the pattern of expression of these 2 genes was region-dependent with Arg-1 maximally expressed in the cortex (about 20-fold increase $v s$ vehicle-treated mice) and IL-10 maximally expressed in the lumbar spinal cords (about a 15-fold increase $v s$ vehicle-treated mice).

Finally, we analyzed whether fingolimod could increase the expression of BDNF, as previously shown in microglial cultures [22]. We found that BDNF mRNA levels were not affected after 2 weeks of fingolimod treatment compared with vehicle-treated $\mathrm{mSOD} 1^{\mathrm{G} 93 \mathrm{~A}}$ mice but, after chronic treatment with fingolimod until the end stage of disease, they were significantly increased in all 3 regions. 


\section{Discussion}

ALS is a progressive, rapidly fatal, and incurable neurodegenerative disease, and thus the identification of new therapeutic approaches is urgently required.

Activation of microglia and infiltration of lymphocytes are involved in the pathogenesis of this disease [5, 14, 29], suggesting the possibility of treating ALS by modulating the immune response. However, the role of the immune system in ALS is very complex and dual in nature, which may well account for the fact that, to date, immunomodulating and immunosuppressant treatments have been partially successful in animal models of the disease, and disappointing overall in patients.

Fingolimod, a potent agonist of the sphingosine-1phosphate receptor, is able to sequester lymphocytes in lymph nodes, to block their trafficking to the target organs, and to increase the proportion of Tregs [30,31]. The latter effect is very relevant to ALS as Tregs play an endogenous neuroprotective function by augmenting, among other mechanisms, the protective potential of microglia in ALS mice [29], and as the number of Tregs is inversely correlated with disease progression both in ALS mice and in patients [12, 28]. Besides its activity on lymphocytes, fingolimod downregulates the production of proinflammatory cytokines and upregulates that of BDNF and glial cell-derived neurotrophic factor in microglial cultures, suggesting that in the CNS the drug might directly promote the neuroprotective effects of microglia [22].

The aim of this study was to evaluate the effects of fingolimod (administered from the onset of symptoms until death) in the widely used $\mathrm{mSOD} 1^{\mathrm{G} 93 \mathrm{~A}}$ ALS mouse model. Our results show, for the first time, that chronic administration of fingolimod reduces the neurological deficits and extends the survival of $\mathrm{mSOD} 1^{\mathrm{G} 93 \mathrm{~A}}$ mice.

The different outcome observed in the neurological evaluations with respect to rotarod test is not surprising. Accelerated rotarod is not only the result of enhanced general locomotor, but also requires motor skill learning [32]: the length of time that mice stay on a rotating rod is a measure of their balance, coordination, physical condition, and motor planning - a range of behaviors that require well-integrated processes much more complex than those analyzed in neuroscore. Furthermore the decline in the accelerating version of the rotarod can be much more affected by fatigue than the spontaneous walking analyzed in neurological tests [33].

Notably, fingolimod treatment was started at symptomatic stage - namely after motor dysfunction onset - which is very important in light of the translation of the results of our study to the clinical setting as the large majority of patients with ALS are affected by the sporadic form of the disease and can be treated only at time of diagnosis. The translation from mice to human patients with ALS has been often disappointing and starting animals treatment before the symptom onset is considered a methodological weakness for the predictive values of preclinical research with ALS mice [34]. Furthermore, fingolimod is already licensed for clinical use in multiple sclerosis, thus further increasing the translational potential of our results.

In addition to the amelioration of the neurological deficits and survival, we observed a modulation of the expression of genes involved in the neuroinflammatory response, favoring a $\mathrm{T} 2$ response.

The doses of fingolimod used in this study $(0.1 \mathrm{mg} / \mathrm{kg}$ and $1 \mathrm{mg} / \mathrm{kg}$ ) were selected on the basis of previous reports showing their effectiveness in animal models of Huntington's disease and Alzheimer's disease [24, 25]. Interestingly, both doses elicited very similar qualitative and quantitative effects in ALS mice, indicating the occurrence of a ceiling effect at doses as low as $0.1 \mathrm{mg} / \mathrm{kg}$. For this reason, we limited the evaluation of the neuroinflammatory parameters to a group treated with the lowest dose of fingolimod $(0.1 \mathrm{mg} / \mathrm{kg})$. The myeloid marker CD11b and transcription factor FoxP3 were analyzed as indicators of microglial activation and Treg functions, respectively. To qualify the type of microglial reactivity, that is, M1 versus M2 response, the expression of a set of genes typically associated with either the M1 (iNOS, IL-1 $\beta$ ), or the M2 (Arg-1, IL-10) state, was investigated. In addition, we analyzed the expression of the neurotrophic factor BDNF, which declined in lumbar spinal cords of ALS mice during the rapidly progressing phase [29], and has been shown to be upregulated by fingolimod [22].

Overall, our results suggest that chronic treatment with fingolimod from symptomatic stage might counteract the T2 to T1 switch, known to occur with the progression of the disease. The increased expression of genes such as Argl, $I l 10$, and Bdnf, and the concomitant decrease in iNOS and IL-1 $\beta$ expression in fingolimod-treated mice at end stage are suggestive of a T2-type immune response, contributing to the better neurological performance and extended survival of fingolimod-treated $\mathrm{mSOD} 1^{\mathrm{G} 93 \mathrm{~A}}$ mice compared with vehicle-treated mice. This interpretation is consistent with the parallel raise in FoxP3 expression induced by fingolimod, suggestive of an increase in the Treg cell populationsupporting the T2 response [28] — in both spinal cord and cerebrum.

The dampened $\mathrm{T} 1$ response, suggested by the reduced iNOS and IL- $1 \beta$ expression, is consistent with the decreased expression of $\mathrm{CD} 11 \mathrm{~b}$ induced by fingolimod. CD11b microglia expression is considered an histological marker for disease progression in animal model of ALS [35], as it is already detectable at clinical onset to further increase at end-stage disease [14]. Inhibition of both iNOS and IL-1 $\beta$ have been proposed as potential therapeutic approach to ALS, as in ALS mice upregulation of iNOS occurs in parallel with motor 
neuron loss [36], and treatment of presymptomatic ALS mice with IL-1 blocker anakinra increases survival without affecting the time of disease onset [37]. M1 microglia-mediated motor neuron death, driven by nuclear factor $\mathrm{kB}$-dependent mechanisms, has been shown in $\mathrm{mSOD} 1^{\mathrm{G} 93 \mathrm{~A}}$ mice and heterozygous inhibition of nuclear factor $\mathrm{kB}$ specifically in the myeloid lineage of $\mathrm{mSOD} 1^{\mathrm{G} 93 \mathrm{~A}}$ mice significantly delayed disease progression and increased survival [4], further supporting the hypothesis that a dampened M1 response might contribute to protective action of fingolimod. Our data are also consistent with recent studies demonstrating that fingolimod is able to modulate microglial inflammatory phenotype both in vitro and in vivo [38], in an animal model of multiple sclerosis [39].

Furthermore, our results suggest that fingolimod treatment might influence $\mathrm{T} 1$ and $\mathrm{T} 2$ responses, with a region-specific pattern.

Regional and temporal differences in neurodegeneration and immune response have been reported in ALS animal models [28, 40, 41], by which the cortex is the less affected and the lumbar spinal cord the earliest and most affected region. These distinct responses have been related to specific patterns of microglial activation and lymphocyte infiltration [28], and the corresponding gradual T2 to T1 phase switch [5]. Consistently, in early symptomatic mSOD $1^{\mathrm{G} 93 \mathrm{~A}}$ mice we found different levels of expression of $\mathrm{CD} 11 \mathrm{~b}$ and FoxP3 in the 3 regions examined (see Table 1).

In chronically treated $\mathrm{mSOD} 1^{\mathrm{G} 93 \mathrm{~A}}$ mice, although iNOS and IL-1 $\beta$ expression was negatively regulated to a similar extent in all 3 brain regions examined, the anti-inflammatory markers Arg-1 and IL-10 showed a rostrocaudal gradient of induction, which was descending for Arg-1 and ascending for IL-10. The different responsiveness to fingolimod was even more pronounced at the earlier time point (i.e., 2 weeks of treatment), when all M1 and M2 genes were downregulated in the lumbar spinal cord, but none of them was affected in the cervical spinal cord, despite a significant increase of FoxP3 mRNA levels. In motor cortex, we found an upregulation of IL- $1 \beta$ counterbalanced by a concomitant upregulation of Arg1 and IL-10, suggestive of an overall effect favouring T2 response.

In conclusion, our data show that fingolimod extends the survival, improves the phenotype and modulates, in a multifaceted way, neuroinflammation in the cortex and spinal cord of ALS mice. Although our study does not demonstrate a causal relation between modulation of neuroinflammation and beneficial effects of fingolimod, it provides - to the best of our knowledge-the first evidence of molecular mechanisms associated with in the drug in a well-characterized ALS mouse model and a convincing preclinical proof of concept of the therapeutic potential of fingolimod in human ALS.
Required Author Forms Disclosure forms provided by the authors are available with the online version of this article.

\section{References}

1. Cleveland DW, Rothstein JD. From Charcot to Lou Gehrig: deciphering selective motor neuron death in ALS. Nat Rev Neurosci 2001;2:806-819.

2. Boillée S, Vande Velde C, Cleveland DW. ALS: a disease of motor neurons and their nonneuronal neighbors. Neuron 2006; 52:39-59.

3. Zhao W, Beers DR, Appel SH. Immune-mediated mechanisms in the pathoprogression of amyotrophic lateral sclerosis. J Neuroimmune Pharmacol. 2013;8:888-899.

4. Frakes AE, Ferraiuolo L, Haidet-Phillips AM, et al. Microglia induce motor neuron death via the classical NF-kB pathway in amyotrophic lateral sclerosis. Neuron 2014;81:1009-1023.

5. Murdock BJ, Bender DE, Segal BM, Feldman EL. The dual roles of immunity in ALS: injury overrides protection. Neurobiol Dis 2015;77:1-12.

6. Zhang R, Gascon R, Miller RG, et al. Evidence for systemic immune system alterations in sporadic amyotrophic lateral sclerosis (sALS). J Neuroimmunol 2005;159: 215-224.

7. Hovden H, Frederiksen JL, Pedersen SW. Immune system alterations in amyotrophic lateral sclerosis. Acta Neurol Scand 2013;128:287-296.

8. Troost D, van den Oord JJ, de Jong JM, Swaab DF. Lymphocytic infiltration in the spinal cord of patients with amyotrophic lateral sclerosis. Clin Neuropathol 1989;8:289-294.

9. Hickey WF. T-lymphocyte entry into the central nervous system. J Neurosci Res 1991;28:254-260.

10. Lewis CA. The neuroinflammatory response in ALS: the roles of microglia and T cells. Neurol Res Int 2012;2012:803701.

11. Engelhardt JI, Tajti J, Appel SH. Lymphocytic infiltrates in the spinal cord in amyotrophic lateral sclerosis. Arch Neurol 1993;50: 30-36.

12. Henkel JS, Beers DR, Wen S, et al. Regulatory T-lymphocytes mediate amyotrophic lateral sclerosis progression and survival. EMBO Mol Med 2013;5:64-79.

13. Hall ED, Oostveen JA, Gurney ME. Relationship of microglial and astrocytic activation to disease onset and progression in a transgenic model of familial ALS. Glia 1998;23:249-256.

14. Alexianu ME, Kozovska M, Appel SH. Immune reactivity in a mouse model of familial ALS correlates with disease progression. Neurology 2001;57:1282-1289.

15. Turner MR, Cagnin A, Turkheimer FE, et al. Evidence of widespread cerebral microglial activation in amyotrophic lateral sclerosis: an [11C](R)-PK11195 positron emission tomography study. Neurobiol Dis 2004;15:601-609.

16. Appel SH, Beers DR, Henkel JS. T cell-microglial dialogue in Parkinson's disease and amyotrophic lateral sclerosis: are we listening? Trends Immunol 2010;31:7-17.

17. Schwartz M, Shechter R. Systemic inflammatory cells fight off neurodegenerative disease. Nat Rev Neurol 2010;6:405-410.

18. Pinschewer DD, Ochsenbein AF, Odermatt B, Brinkmann V, Hengartner H, Zinkernagel RM. FTY720 immunosuppression impairs effector T cell peripheral homing without affecting induction, expansion, and memory. J Immunol 2000;164:5761-5770.

19. Kahan BD. FTY720: from bench to bedside. Transplant Proc 2004; 36(2 Suppl.):531S-543S.

20. Serpero LD, Filaci G, Parodi A, et al. Fingolimod modulates peripheral effector and regulatory $\mathrm{T}$ cells in MS patients. $\mathrm{J}$ Neuroimmune Pharmacol 2013;8:1106-1113. 
21. Miron VE, Schubart A, Antel JP. Central nervous system-directed effects of FTY720 (fingolimod). J Neurol Sci 2008;274:13-17.

22. Noda H, Takeuchi H, Mizuno T, Suzumura A. Fingolimod phosphate promotes the neuroprotective effects of microglia. J Neuroimmunol 2013;256:13-18.

23. Di Menna L, Molinaro G, Di Nuzzo L, et al. Fingolimod protects cultured cortical neurons against excitotoxic death. Pharmacol Res 2013;67:1-9.

24. Di Pardo A, Amico E, Favellato M, et al. FTY720 (fingolimod) is a neuroprotective and disease-modifying agent in cellular and mouse models of Huntington disease. Hum Mol Genet 2014;23:22512265.

25. Fukumoto K, Mizoguchi H, Takeuchi H, et al. Fingolimod increases brain-derived neurotrophic factor levels and ameliorates amyloid $\beta$-induced memory impairment. Behav Brain Res 2014;15;268:88-93.

26. Scott S, Kranz JE, Cole J, et al. Design, power, and interpretation of studies in the standard murine model of ALS. Amyotroph Lateral Scler 2008;9:4-15.

27. Ludolph AC, Bendotti C, Blaugrund E, et al. Guidelines for preclinical animal research in ALS/MND: a consensus meeting. Amyotroph Lateral Scler 2010;11:38-45.

28. Beers DR, Henkel JS, Zhao W, et al. Endogenous regulatory T lymphocytes ameliorate amyotrophic lateral sclerosis in mice and correlate with disease progression in patients with amyotrophic lateral sclerosis. Brain 2011; 134:1293-1314.

29. Beers DR, Henkel JS, Zhao W, Wang J, Appel SH. CD4+ T cells support glial neuroprotection, slow disease progression, and modify glial morphology in an animal model of inherited ALS. Proc Natl Acad Sci U S A 2008;105:15558-15563.

30. Mandala S, Hajdu R, Bergstrom J, et al. Alteration of lymphocyte trafficking by sphingosine-1-phosphate receptor agonists. Science 2002;296:346-349.

31. Brinkmann V, Billich A, Baumruker T, et al. Fingolimod (FTY720): discovery and development of an oral drug to treat multiple sclerosis. Nat Rev Drug Discov 2010;9:883-897.
32. Buitrago MM, Schulz JB, Dichgans J, Luft AR. Short and longterm motor learning in an accelerated rotarod training paradigm. Neurobiol Learn Mem 2004;81:211-216.

33. Brooks SP, Dunnett SB. Tests to assess motor phenotype in mice: a user's guide. Nat Rev Neurosci 2009;10:519-529.

34. Benatar M. Lost in translation: treatment trials in the SOD1 mouse and in Human ALS. Neurobiol Dis 2007; 26:1-13.

35. Graber DJ, Hickey WF, Harris BT. Progressive changes in microglia and macrophages in spinal cord and peripheral nerve in the transgenic rat model of amyotrophic lateral sclerosis. J Neuroinflammation 2010;7:8.

36. Lee J, Ryu H, Kowall NW. Differential regulation of neuronal and inducible nitric oxide synthase (NOS) in the spinal cord of mutant SOD1 (G93A) ALS mice. Biochem Biophys Res Commun 2009; 387:202-206.

37. Meissner F, Molawi K, Zychlinsky A. Mutant SOD1 induced IL-1 $\beta$ accelerates ALS pathogenesis. Proc Natl Acad Sci U S A 2010;107: 13046-13050.

38. Cipriani R, Chara JC, Rodríguez-Antigüedad A, Matute C. FTY720 attenuates excitotoxicity and neuroinflammation. J Neuroinflammation 2015;12:86.

39. Airas L, Dickens AM, Elo P, et al. In vivo PET imaging demonstrates diminished microglial activation after fingolimod treatment in an animal model of multiple sclerosis. J Nucl Med 2015;56:305310 .

40. Lewis KE, Rasmussen AL, Bennett W, et al. Microglia and motor neurons during disease progression in the SOD1G93A mouse model of amyotrophic lateral sclerosis: changes in arginase 1 and inducible nitric oxide synthase. J Neuroinflammation 2014;11:55.

41. Nikodemova M, Small AL, Smith SM, Mitchell GS, Watters JJ. Spinal but not cortical microglia acquire an atypical phenotype with high VEGF, galectin-3 and osteopontin, and blunted inflammatory responses in ALS rats. Neurobiol Dis 2014;69:43-53. 